\title{
SPECTRAL DISTRIBUTION STUDIES WITH MODIFIED KUO-BROWN INTERACTION IN THE UPPER HALF OF THE fp SHELL
}

\author{
S. Choubey and K.Kar \\ Saha Institute of Nuclear Physics \\ Block - AF, Sector I, Bidhan Nagar \\ Calcutta 700 064, India \\ J.M.G. Gomez \\ Departamento de Fisica Atomica, Molecular y Nuclear \\ Facultad de Ciencias Fisicas, Universidad Complutense de Madrid \\ E-28040 Madrid, Spain \\ V.R. Manfredi \\ Dipartimento di Fisica "G. Galilei", Università di Padova \\ Istituto Nazionale di Fisica Nucleare - Sezione di Padova \\ via Marzolo 8, I-35131, Padova, Italy \\ Received:
}

\begin{abstract}
The spectral distribution method with modified Kuo-Brown interaction is extended to the study of the upper half as well as to odd-A nuclei, of the fp shell. The calculations show similar success to that obtained for the lower half.
\end{abstract}


Microscopic calculations in the fp-shell involving all four valence orbits $f_{7 / 2}, f_{5 / 2}, p_{3 / 2}$ and $p_{1 / 2}$ have been actively persued in the last few years. Among the residual two body interactions used for this purpose modified Kuo-Brown (KB3) has shown remarkable success for the lighter fp-shell nuclei in the full shell model diagonalisation calculations [1,2] as well as in the Monte-Carlo shell model studies [3] for both spectra as well as transition strengths. Spectral distribution theory, originally constructed to reproduce the global features of level densities and transition strengths [4] is seen also to reproduce binding energies, low lying spectra and transition strengths equally well. These studies were performed in detail for the sd-shell $[4,5]$ and in some cases for the fp-shell nuclei [6]. Recently such calculations were carried out with the KB3 interaction in the lower half of the fp-shell [7] with reasonable success. Some of the studies are motivated by applications to nuclear astrophysics, such as the problem of calculating the electron capture/beta decay rates for supernova and presupernova evolution and $\mathrm{r}$ and s process nucleosynthesis. Therefore one feels the need to extend the calculations to the upper half of the fp-shell as many important nuclei for such applications have $\mathrm{A}>60$. We should note here that KB3 was constructed to improve the spectroscopy of nuclei at the beginning of the fp-shell and in the absence of full diagonalisation the success of KB3 for heavier fp-shell nuclei is still uncertain. But KB3 correctly modifies the diagonal matrix elements of the 2-body residual interaction and as spectral distribution theory relies on the lower order moments of the Hamiltonian in the many body shell model spaces, which depend more crucially on the diagonal matrix elements than on the nondiagonal ones, we consider it interesting to apply the KB3 
interaction for the spectral distribution studies in the upper half of the fpshell. In this report we study spectra, occupancies and sum rule strengths of transition operators, using the KB3 interaction in the upper half of the fp-shell. Here we incorporate the corrections coming from the third and the fourth moments of the Hamiltonian in terms of the nonzero skewness $\left(\gamma_{1}\right)$ and excess $\left(\gamma_{2}\right)$. We shall see that compared to the earlier spectral distribution results [6], the agreement of the binding energies with the experimental values, particularly for nuclei with $\mathrm{A}>70$, is considerably improved. We also apply KB3 interaction to the odd-A nuclei in the fp-shell for the first time and see that after taking into account corrections from nonzero $\left(\gamma_{1}, \gamma_{2}\right)$ the agreement with experimental data is satisfactory.

Spectral distribution theory gives smoothed fluctuation free forms for the density of states as a function of energy, which in large shell model spaces asymptotically go towards gaussians. The formal derivation of the result uses the central limit theorem (CLT) for the one body Hamiltonian $(\mathrm{H}(1))$ and extends that to the two body Hamiltonian $(\mathrm{H}(2))$, defining an ensemble of Hamiltonians and averaging the moments $\left\langle H^{p}(2)>, \mathrm{p}=1,2, \ldots\right.$ over the ensemble [8]. For the 2-body Hamiltonian the ensemble averaged results in many particle space follow from the dominance of binary correlations, as elegantly demonstrated by Mon and French [8]. Spectral distribution also gives polynomial expansions for the expectation values of operators in terms of energy where only the first two terms in the expansion contribute in the CLT limit [4]. Partitioning the shell model space of $\mathrm{m}$ valence particles in $\mathrm{N}$ single particle states according to configuration and isospin $((\tilde{m}, T)$ spaces where $\tilde{m}=m_{1}, m_{2}, \ldots m_{l}$ are the number of particles in the 1 orbits) and the 
use of gaussian or gaussian modified by Cornish-Fisher expansion around it for the density of states, increases the predictability of the method. To find the ground state energy $\bar{E}_{g}$ one inverts the equation

$$
\sum_{\tilde{m}} \int_{-\infty}^{\bar{E}_{g}} I_{\tilde{m}, T}(E) d E=d_{0} / 2
$$

where $I_{\tilde{m}, T}$ is the gaussian density of states in the $(\tilde{m}, T)$ spaces normalised to $\mathrm{d}(\tilde{m}, T)$, the dimensionality of the configuration isospin space, and $d_{0}$ $(=(2 \mathrm{~J}+1))$ is the degeneracy of the ground state with spin J. Thus one integrates the area below the gaussian configuration densities until the area becomes equal to half the ground state degeneracy. This energy value is the predicted ground state energy and the method is called the Ratcliff procedure [9]. As done earlier for the sd-shell [10] and the lower half of the fp-shell [7], we improve the predictions by incorporating the corrections from nonzero skewness $\left(\gamma_{1}\right)$ and excess $\left(\gamma_{2}\right)$ by using the Cornish-Fisher expansion which gives

$$
x=y+\frac{\gamma_{1}}{6}\left(y^{2}-1\right)+\frac{\gamma_{2}}{24}\left(y^{3}-3 y\right)-\frac{\gamma_{1}^{2}}{36}\left(2 y^{3}-5 y\right)
$$

where $y$ is the normalised energy $(y=(E-\epsilon) / \sigma, \epsilon$ is the centroid and $\sigma$ is the width) before the correction and $x$ is the value after it. For the centroids and widths we use the values in $(\mathrm{m}, \mathrm{T})$ spaces. For $\gamma_{1}$ and $\gamma_{2}$, ideally one should use the values in $(\mathrm{m}, \mathrm{T})$ spaces calculating the third and the fourth moments of the $(1+2)$-body Hamiltonian. But as at present the spectral distribution method (SDM) codes can calculate them only in the scalar (m) spaces. We use a phenomenological correction term for the excess using $\gamma_{2}=\gamma_{2}(m)+a m+b m^{2}$ where the values of 'a ' and 'b' are obtained through a best fit. In the upper half of the fp shell, we find that a parametrised 
dependence of $\gamma_{2}$ on isospin makes little improvement in the agreement of the calculated binding energies with the experimental ones in contrast to the lower half. As the $\gamma_{1}$ corrections are very small, we keep the $\gamma_{1}(m)$ unchanged. We calculate the binding energies at fixed configurations before and after the corrections and give in Table 1 the difference between the predicted and the experimental values given by $\mathrm{DIFF}=$ (calculated binding energy - experimental binding energy) $\mathrm{MeV}$. The binding energies are taken to be positive in agreement with the convention used by experimentalists. We also list the corresponding values obtained by Haq and Parikh [6] to show that by using the KB3 interaction and by incorporating the $\left(\gamma_{1}, \gamma_{2}\right)$ corrections, we obtain considerable improvement over the earlier calculations done by them, where they utilise scalar isospin moments with excited state correction and use the MHW2 interaction. For the odd-A nuclei the best predictions are obtained using $a=0.006$ and $b=-0.00029$ and the RMS deviation of the calculated values from the experimental values is $1.22 \mathrm{MeV}$. For the odd-odd nuclei the best predictions are obtained using $a=0.005$ and $b=-0.00028$ and the RMS deviation of the calculated values from the experimental values is 1.34 MeV. For the even-even nuclei the best predictions are obtained using $a=0.007$ and $b=-0.00031$ and the RMS deviation of the calculated values from the experimental values is $1.77 \mathrm{MeV}$. One observes that correction of $\gamma_{2}$ separately for the odd-A, even-even and odd-odd nuclei brings the values closer to experimental ones, as this way one is able to take account of the ground state pairing effects to some extent.

There are indications from experimental pick-up/stripping reaction data that the orbit $1 g_{9 / 2}$ starts picking up neutrons when the neutron number 
of the nuclei goes close to 40 [11]. We have constrained our calculations to the four fp-shell orbits and this may be one of the reasons for the somewhat larger deviations of the SDM values from the experimental binding energies for neutrons almost filling the shell. We note the considerable improvement one achieves by indroducing the $\left(\gamma_{1}, \gamma_{2}\right)$ corrections for these nuclei compared to the Haq-Parikh values as seen in Table 1. But we feel future studies for $\mathrm{A}=70$ and beyond should include excitations of particles to the $1 g_{9 / 2}$ orbit.

To understand the global properties of the KB3 interaction one should study the centroids, widths and its correlation coefficient with other typical interactions in the fp-shell evaluated in the $(\mathrm{m}, \mathrm{T})$ spaces. For the sake of comparison, we choose the MHW2 interaction [12]. We give the typical example of $m=24$ and $m=28$ with their two extreme isospin values,but the behavior at other particle numbers and isospins is very similar. For $\mathrm{m}=24$, the centroids for $\mathrm{T}=0$ and $\mathrm{T}=8$ are $-243.89 \mathrm{MeV}$ and $-193.21 \mathrm{MeV}$ for KB3 and -229.80 $\mathrm{MeV}$ and $-186.49 \mathrm{MeV}$ for MHW2 respectively. For $\mathrm{m}=28$ the centroids for $\mathrm{T}=0$ and 6 are $-304.12 \mathrm{MeV}$ and $-274.55 \mathrm{MeV}$ for KB3 and $-285.25 \mathrm{MeV}$ and -259.98 MeV for MHW2 respectively.As essentially KB3 differs from MHW2 in the diagonal two-body matrix elements, one understands the differences in the centroids observed. The widths for the MHW2 interaction are seen to be different from the KB3 interaction by a factor between 0.75 to 0.90 in the upper half. For example for $\mathrm{m}=24$ and $\mathrm{T}=0$ the width for KB3 is 14.89 $\mathrm{MeV}$, whereas for MHW2 it is $13.09 \mathrm{MeV}$. As the nondiagonal elements for the two interactions are almost identical, the correlation coefficient between the two interactions, which has the centroids subtracted out, is always close to one throughout the shell. Typically for $m=28$ it varies between 0.992 to 
0.989 .

Spectral distribution gives a polynomial expansion in energy for the expectation values of operators where the terms beyond the first two in the expansion are inhibited by CLT. Explicitly for the operator $K$ in the $(\mathrm{m}, \mathrm{T})$ space, the CLT form for its expectation value at energy E is,

$$
K(E ; m, T)=\langle m, T|K| m, T\rangle+\zeta_{K-H}(m, T) \sigma_{K}(m, T) \frac{E-E_{c}(m, T)}{\sigma(m, T)}
$$

where $\sigma_{K}(m, T)$ is the width of the operator $K$ in the space $(\mathrm{m}, \mathrm{T})$ and $\zeta_{H-K}$ is the correlation coefficient between $K$ and $H$ in the space $(\mathrm{m}, \mathrm{T}) . E_{c}(\mathrm{~m}, \mathrm{~T})$ and $\sigma(m, T)$ are the centroid and width of the Hamiltonian in the same space. Taking $K=n_{s}$, the number operator for the orbit s, one can find the occupancy of the orbit s. One can also obtain such expansions for the expectation values in specific configuration isospin $(\tilde{m}, \mathrm{~T})$ spaces and then average over all configurations. Table 2 shows such configuration averaged occupancies calculated for the 4 orbits at the ground state energies for some typical values of valence nucleons and isospins. These results are also available for all valence nucleon numbers and isospins. But we mention here that the SDM values given are averaged over all J states. Occupancies through shell model calculations or from experimental pick-up/stripping sum rules are for spaces with fixed J. Ideally one needs to do a J-projection of the SDM value for proper comparisons. A detailed comparison of such occupancies by SDM and the shell model in the fp-shell, is, in our opinion, of great interest.

We also use for $K$ the Gamow-Teller (GT) sum rule operator i.e. $K=$ $\left(O^{11} \times O^{11}\right)^{00}$ with $O^{11}$ being the GT transition operator, a vector in both $\mathrm{J}$ and T. Then for states with isospin zero, we obtain through Eq.(3) the 
sum rule strength for the Gamow-Teller transition to all final states. In Table 3 we give sum rule strengths for self conjugate nuclei (i.e. with $\mathrm{N}=\mathrm{Z}$ ) with valence particles $22,24,32$ and 34 . The table explicitly shows how the correlation of $K$ with $H$ changes the sum rule strengths. As the correlation coefficient between $K$ and $H$ is very small, the inclusion of the second term brings about a decrease of less than $3 \%$. These sum rule estimates of the GT strength are useful for the calculations of the electron capture rates on these nuclei during the collapse phase of the supernova or the beta decay rates for the presupernova evolution [13].

One can also use such sum rule estimates for other one body interaction operators also. These calculations can be extented to nuclei with nonzero ground state isospin. The success of the SDM in reproducing average energies as well as transition strengths gives one a method of evaluating many structural properties of nuclei with many valence nucleons in active orbits, avoids explicit diagonalisation and is also useful for astrophysical applications.

We thank S. Sarkar for his help and for many useful discussions; V. K. B. Kota for supplying us some of the SDM programs. This work has been partially supported by the Secific Project "Milano 41" and DGES Project No. PB96-0604. 


\section{REFERENCES}

[1] E. Caurier, A.P. Zuker, A. Poves and G. Martinez-Pinedo, Phys. Rev. C 50, 225 (1994); E. Caurier, G. Martinez-Pinedo, A. Poves and A.P. Zuker, Phys. Rev. C 52, R1736 (1995).

[2] G. Martinez-Pinedo, A. P. Zuker, A. Poves and E. Caurier, Phys. Rev. C 55, 187 (1997).

[3] S.E. Koonin, D.J. Dean and K. Langanke, Phys. Rep. 278, 1 (1996); K. Langanke, D. J. Dean, P. B. Radha, Y. Allhassid and S. E. Koonin, Phys. Rev. C 52, 718 (1995).

[4] J.B. French in "Nuclear Structure", 1967, edited by A. Hossain, Harunal-Rashid and M. Islam, (North-Holland, Amsterdam); J.P. Draayer, J.B. French and S.S.M. Wong, Ann. Phys. (N.Y.) 106, 472 (1977); J.B. French and V.K.B. Kota Annu. Rev. Nucl. Part. Sci. 32, 35 (1982).

[5] V.K.B. Kota and K. Kar, University of Rochester, Laboratory Report No. UR-1058, 1988; Pramana 32, 647 (1989).

[6] R.U. Haq and J.C. Parikh, Nucl. Phys. A273, 410 (1976); V.K.B. Kota and V. Potbhare, Nucl. Phys. A331, 93 (1979).

[7] K. Kar, S. Sarkar, J.M.G Gomez, V.R. Manfredi and L. Salanich, Phys. Rev. C 55, 1260 (1997).

[8] K. K. Mon and J.B. French, Ann. Phys. 95, 90 (1975).

[9] K. F. Ratcliff, Phys. Rev. C 3, 117 (1971).

[10] S. Sarkar, K. Kar and V.K.B. Kota, Phys. Rev. C 36, 2700 (1987).

[11] G. J. Matthews, S. D. Bloom, G. M. Fuller and J. N. Bahcall, Phys. Rev. C 32, 796 (1985). 
[12] J. B. McGrory, B. H. Wildenthal and E. C. Halbert, Phys. Rev C 2, 186 (1970).

[13] K. Kar, A. Ray and S. Sarkar, Astrophys. J. 434, 662 (1994) 


\section{Table 1}

The difference, DIFF in MeV, between the experimental and the calculated binding energies. Column $\bar{A}, \bar{B}$ and $\bar{C}$ give the difference corresponding to the value calculated by Ratcliff procedure in $(\tilde{m}, T)$ space, by Ratcliff procedure with $\left(\gamma_{1}(m), \gamma_{2}(m)\right)$ correction and by Ratcliff with $\left(\gamma_{1}(m, T)\right.$, $\left.\gamma_{2}(m, T)\right)$ correction respectively. The last column gives DIFF corresponding to earlier SDM predictions with excited state correction using the MHW2 interaction [6].

\begin{tabular}{ccccc}
\hline Nucleus & \multicolumn{3}{c}{ DIFF } & DIFF \\
& \multicolumn{3}{c}{ with KB3 } & with \\
& $\bar{A}$ & $\bar{B}$ & $\bar{C}$ & MHW2 \\
\hline${ }^{61} \mathrm{Zn}$ & 18.3 & -0.6 & -0.8 & 4.0 \\
${ }^{61} \mathrm{Cu}$ & 18.1 & -0.5 & -0.7 & 2.0 \\
${ }^{61} \mathrm{Ni}$ & 17.6 & 0.2 & 0.1 & 0.9 \\
${ }^{61} \mathrm{Co}$ & 15.1 & 1.1 & 0.9 & 1.7 \\
${ }^{61} \mathrm{Fe}$ & 14.4 & 2.5 & 2.4 & 3.1 \\
${ }^{63} \mathrm{Ga}$ & 19.2 & 1.1 & -0.6 & 2.5 \\
${ }^{63} \mathrm{Zn}$ & 19.5 & 1.5 & -0.1 & 3.0 \\
${ }^{63} \mathrm{Cu}$ & 18.0 & 1.2 & -0.4 & 0.5 \\
${ }^{63} \mathrm{Ni}$ & 17.7 & 2.1 & 0.7 & 1.8 \\
${ }^{63} \mathrm{Co}$ & 16.6 & 5.1 & 4.0 & 1.4 \\
${ }^{65} \mathrm{Ge}$ & 19.9 & 3.1 & 0.1 & 0.6 \\
\hline
\end{tabular}


Table 1 (contd.)

\begin{tabular}{|c|c|c|c|c|}
\hline \multirow[t]{2}{*}{ Nucleus } & \multicolumn{3}{|c|}{$\begin{array}{c}\text { DIFF } \\
\text { with KB3 }\end{array}$} & \multirow{2}{*}{$\begin{array}{c}\text { DIFF } \\
\text { with } \\
\text { MHW2 }\end{array}$} \\
\hline & $\bar{A}$ & $\bar{B}$ & $\bar{C}$ & \\
\hline${ }^{65} G a$ & 19.6 & 2.9 & -0.1 & 0.4 \\
\hline${ }^{65} Z n$ & 17.8 & 2.6 & -0.1 & -3.9 \\
\hline${ }^{65} \mathrm{Cu}$ & 15.9 & 2.1 & -0.4 & -5.2 \\
\hline${ }^{65} \mathrm{Ni}$ & 12.6 & 1.8 & -0.2 & -8.4 \\
\hline${ }^{67} G a$ & 17.7 & 3.9 & 0.0 & -4.6 \\
\hline${ }^{67} Z n$ & 14.6 & 3.0 & -0.3 & -10.2 \\
\hline${ }^{67} \mathrm{Cu}$ & 11.9 & 2.1 & -0.7 & -10.9 \\
\hline${ }^{69} \mathrm{Ge}$ & 15.9 & 4.8 & 0.5 & -9.6 \\
\hline${ }^{69} G a$ & 13.7 & 3.7 & -0.1 & -13.8 \\
\hline${ }^{69} Z n$ & 11.6 & 3.2 & -0.1 & -15.0 \\
\hline${ }^{69} \mathrm{Cu}$ & 4.6 & -0.4 & -2.3 & 22.2 \\
\hline${ }^{71} \mathrm{Se}$ & 15.7 & 6.3 & 1.8 & -12.4 \\
\hline${ }^{71} A s$ & 13.9 & 5.4 & 1.3 & -16.6 \\
\hline${ }^{62} Z n$ & 20.2 & -0.4 & 0.1 & 2.1 \\
\hline${ }^{62} N i$ & 18.2 & 0.2 & 0.7 & 0.1 \\
\hline${ }^{64} G e$ & 19.9 & 1.1 & 0.0 & 2.0 \\
\hline${ }^{64} Z n$ & 20.1 & 1.3 & 0.2 & -0.3 \\
\hline${ }^{64} N i$ & 15.9 & 1.2 & 0.3 & -3.7 \\
\hline
\end{tabular}


Table 1 (contd.)

\begin{tabular}{|c|c|c|c|c|}
\hline \multirow[t]{2}{*}{ Nucleus } & \multicolumn{3}{|c|}{$\begin{array}{c}\text { DIFF } \\
\text { with KB3 }\end{array}$} & \multirow{2}{*}{$\begin{array}{c}\text { DIFF } \\
\text { with } \\
\text { MHW2 }\end{array}$} \\
\hline & $\bar{A}$ & $\bar{B}$ & $\bar{C}$ & \\
\hline${ }^{66} \mathrm{Ge}$ & 21.2 & 3.3 & 0.5 & 0.7 \\
\hline${ }^{66} Z n$ & 18.2 & 2.5 & 0.1 & -5.2 \\
\hline${ }^{66} \mathrm{Ni}$ & 12.1 & 1.4 & -0.3 & -8.7 \\
\hline${ }^{68} \mathrm{Ge}$ & 19.3 & 4.3 & 0.4 & -4.3 \\
\hline${ }^{68} Z n$ & 14.1 & 2.7 & -0.3 & -12.8 \\
\hline${ }^{72} K r$ & 8.6 & -0.7 & -5.0 & -20.2 \\
\hline${ }^{72} \mathrm{Se}$ & 15.0 & 5.7 & 1.4 & -14.5 \\
\hline${ }^{74} K r$ & 5.0 & -1.4 & -4.8 & -25.9 \\
\hline${ }^{62} \mathrm{Cu}$ & 19.9 & 1.6 & 1.1 & 4.9 \\
\hline${ }^{62} \mathrm{Co}$ & 15.4 & 2.7 & 0.8 & 1.5 \\
\hline${ }^{64} \mathrm{Ga}$ & 23.3 & 3.8 & -0.9 & 5.6 \\
\hline${ }^{64} \mathrm{Cu}$ & 18.8 & 3.0 & -0.8 & 1.1 \\
\hline${ }^{64} \mathrm{Co}$ & 14.1 & 3.7 & 1.2 & 2.4 \\
\hline${ }^{66} \mathrm{Ga}$ & 22.7 & 5.5 & -0.3 & 2.2 \\
\hline${ }^{66} \mathrm{Cu}$ & 15.9 & 3.7 & -0.4 & -5.0 \\
\hline${ }^{68} \mathrm{Ga}$ & 17.8 & 5.5 & 0.0 & -6.3 \\
\hline${ }^{68} \mathrm{Cu}$ & 10.8 & 3.0 & -0.5 & -14.1 \\
\hline${ }^{74} \mathrm{Br}$ & 14.0 & 7.9 & 3.8 & -18.3 \\
\hline
\end{tabular}


Table 2

Calculated occupancies by SDM for the fp-shell nuclei in the upper half.

\begin{tabular}{ccccccc}
\hline \multirow{2}{*}{$\begin{array}{c}\text { Atomic } \\
\text { Number }\end{array}$} & $\begin{array}{c}\text { Number of } \\
\text { valence } \\
\text { particles }\end{array}$ & Isospin & $f_{7 / 2}$ & $f_{5 / 2}$ & $p_{3 / 2}$ & $p_{1 / 2}$ \\
\hline \multirow{2}{*}{64} & 24 & 0 & 14.59 & 2.41 & 5.20 & 1.81 \\
& & 4 & 14.40 & 3.24 & 4.58 & 1.77 \\
& & & & & & \\
69 & 29 & 5 & 15.38 & 4.60 & 6.44 & 2.59 \\
& & 11 & 15.15 & 6.19 & 5.38 & 2.27 \\
& & & & & & \\
74 & 34 & 1 & 15.96 & 6.62 & 7.83 & 3.60 \\
& & 2 & 15.00 & 3.00 & 4.00 & 2.00 \\
& & & & & & \\
\hline
\end{tabular}




\section{Table 3}

The sum rule strength for Gamow - Teller (GT) transition for $\mathrm{T}=0$ nuclei in $f p$ shell with KB3 interaction by spectral distribution using Eq.(3) which includes terms up to CLT(column B). Column A gives values with only the first term of Eq.(3).

\begin{tabular}{ccc}
\multirow{2}{*}{$\begin{array}{c}\text { no. of } \\
\text { valence }\end{array}$} & \multicolumn{2}{c}{ GT sum rule strength } \\
\cline { 2 - 3 } particles & $\mathrm{A}$ & $\mathrm{B}$ \\
\hline 22 & 14.89 & 14.46 \\
24 & 14.44 & 14.03 \\
32 & 9.62 & 9.44 \\
34 & 7.67 & 7.56 \\
\hline
\end{tabular}

